\title{
CONCEPTS
}

\section{Triaging Multiple Victims in an Avalanche Setting: The Avalanche Survival Optimizing Rescue Triage Algorithmic Approach}

\author{
Lee B. Bogle, MD; Jeff J. Boyd, MBBS, UIAGM; Kyle A. McLaughlin, CCFP (EM) \\ From the University of Calgary, Calgary, AB, Canada (L.B. Bogle); Mineral Springs Hospital, Banff, AB, Canada (J.J. Boyd); and the \\ Department of Emergency Medicine, University of Calgary, Calgary, AB, Canada (K.A. McLaughlin)
}

\begin{abstract}
As winter backcountry activity increases, so does exposure to avalanche danger. A complicated situation arises when multiple victims are caught in an avalanche and where medical and other rescue demands overwhelm resources in the field. These mass casualty incidents carry a high risk of morbidity and mortality, and there is no recommended approach to patient care specific to this setting other than basic first aid principles. The literature is limited with regard to triaging systems applicable to avalanche incidents. In conjunction with the development of an electronic avalanche rescue training module by the Canadian Avalanche Association, we have designed the Avalanche Survival Optimizing Rescue Triage algorithm to address the triaging of multiple avalanche victims to optimize survival and disposition decisions.
\end{abstract}

Key words: mass casualty incident, triage, avalanche, mountain rescue, AvSORT

\section{Introduction}

Avalanche fatalities have been increasing over time in North America, with a 10-season average of 41 deaths/ year between 1998-1999 and 2007-2008. ${ }^{1}$ This correlates with the increase in backcountry skiing, snowboarding, and snowmobiling. ${ }^{2,3}$ Injuries are diverse and may range from asphyxia to traumatic injuries (eg, spinal fractures) and to exposure injuries (eg, hypothermia). ${ }^{4}$ Currently, it is common for extricated avalanche victims to be treated according to standard first aid principles, such as evaluating and treating the $\mathrm{ABCs}$ (airway, breathing, and circulation). Additionally, an avalanche resuscitation algorithm has been developed. ${ }^{5}$ Whereas these can help guide the management of individual patients, they are not designed as triage tools for multiple victims.

Mass casualty incident is a term that may be used in circumstances when multiple victims place an excessive demand on finite resources. ${ }^{6}$ Mass casualties in an avalanche incident not uncommonly overwhelm first responders due to limited manpower in remote settings and the time necessary to locate, dig out, and extricate buried

Corresponding author: Lee B. Bogle, MD, University of Calgary, Calgary, AB, Canada (e-mail: el.bogle@gmail.com). victims. ${ }^{7-10}$ In such incidents, triage needs to rapidly identify those that will benefit from limited resources and may only allow simple lifesaving interventions, such as opening an airway or stopping major bleeding. ${ }^{9,11}$ Traditional cardiopulmonary resuscitation may not be an optimal use of resources, ${ }^{12}$ notably while other potential survivors remain buried.

The hospital disposition of extricated victims should be determined, wherever possible, with those minimally injured being sent to the nearest health care facility and those more severely injured ideally being transported to designated trauma centers. ${ }^{13,14}$

Avalanche rescues entail unique factors that necessitate a distinct triage system. Our goal was to develop a triage tool to guide first-response avalanche rescuers in the management of avalanche incidents when initial resources are overwhelmed by mass casualties. By following a simple algorithmic approach to victim assessment, victims may receive simple lifesaving measures and be stratified for evacuation to the most appropriate facility.

\section{Concept development}

The existing mass casualty triage systems Simple Triage and Rapid Treatment (START), Care-Flight, and 
Triage Sieve were evaluated for applicability to avalanche rescue.$^{8,15-17}$ A literature search was performed using PubMed, EMBASE, and Google Scholar using the search term "avalanche." Broad Internet searches using Google and hand-searching augmented the literature search. This project was undertaken in conjunction with the development of an electronic avalanche rescue training module by the Canadian Avalanche Association. ${ }^{10,18}$

Triage literally means "to sort."19 A mass casualty incident is typified by rescuers and resources being overwhelmed, and the general principle of a mass casualty triage system is to prioritize victims to promote the greatest survival benefit for the greatest number. ${ }^{6}$ The prompt identification of severely injured persons that may benefit from simple interventions, given the limited resources, is particularly essential. Several triage systems exist for mass casualty incidents. START, Care-Flight, and Triage Sieve use an algorithmic approach to stratify patients based on readily observed characteristics such as the ability to walk, respiration status, the ability of a victim to obey a command, and capillary refill. Based on these factors, patients are assigned to immediate, urgent, delayed, or unsalvageable categories. When the individual criteria of these triage systems were analyzed in a retrospective study, it was found that a reduction in the motor component of the Glasgow Coma Score (GCS) below 6 or a systolic blood pressure less than $80 \mathrm{~mm} \mathrm{Hg}$ were the most sensitive predictors of severe injury. ${ }^{11} \mathrm{An}$ inability to obey a simple command reflects a motor component of the GCS score of less than $6 .^{20}$ The absence of a radial pulse is considered indicative of a systolic blood pressure of less than $80 \mathrm{~mm} \mathrm{Hg}^{20}$ and has been proposed as an alternative for slowed capillary refill in mass casualty incidents. ${ }^{17}$ Whereas these triage systems are useful in the general prehospital setting, they are not designed for the specific and austere environment of avalanche rescue.

Overtriage for hospital disposition is the situation where patients with relatively minor injuries are assigned to higher-level trauma centers incorrectly, and a rate of up to $50 \%$ is acceptable in a mass casualty setting to prevent missing any serious injuries. ${ }^{13} \mathrm{Un}$ dertriage is the situation where those who are seriously injured are not appropriately triaged to the severity of their injuries and are thus sent to the nearest hospital instead of a trauma center. Undertriage rates of up to $5 \%$ are acceptable. ${ }^{13}$

An avalanche resuscitation algorithm, derived from original research and adopted by consensus among expert rescue physicians of the Medical Commission of the International Commission for Alpine Rescue (ICAR MedCom), has been developed ${ }^{5,21}$ (Figure 1).
However, it is designed for organized rescue teams, which, in Europe, usually include specialist physicians with advanced life support (ALS) equipment and that respond rapidly due to the widespread cellular phone coverage and short helicopter flight times. ${ }^{22}$ This rapid and skilled response, although ideal, rarely applies to first responders in North America who may have very limited equipment, may lack ALS training, and may be overwhelmed by mass casualties. The ICAR MedCom algorithm focuses on the management of individual victims and is not a triage tool.

In developing our proposed mass casualty algorithm, several factors were taken into account. The algorithm would need to promptly identify survivable and nonsurvivable injuries under the resource-strained circumstances, then divide the potential survivors into those requiring immediate, urgent, or delayed treatment when resources allow.

The avalanche literature describes asphyxia as the cause of death in the majority of fatalities as well as a spectrum of lethal and nonlethal mechanical trauma that may range in incidence from 5\% to $32 \%^{4,23-25}$ (Table). Asphyxia is due to avalanche debris obstructing the airway, from mechanical chest compression, and rebreathing expired air resulting in hypercapnia and hypoxia. $^{26,27}$ The presence of an "air pocket, defined as any space surrounding the mouth and the nose, no matter how small, with a patent airway," is necessary for prolonged survival from burial. ${ }^{5,28}$ Rescue diggers should always search for this air pocket. In victims buried for longer than 35 minutes, the absence of an air pocket foretells death. ${ }^{29}$

\section{The AvSORT algorithm}

Taking into account the above factors, we propose the following Avalanche Survival Optimizing Rescue Triage (AvSORT) algorithm for mass casualties when resources are overwhelmed (Figure 2). We have retained general management concepts from existent mass casualty triage systems but have incorporated key elements specific to avalanche rescue. The inclusion of a step to determine "obvious fatal trauma" is deemed necessary due to the mechanical injury that can occur during an avalanche. Though it relies on the judgment of the first responder as to what is obviously fatal, it could allow rescuers to reallocate precious time to victims who could be saved. After 35 minutes of burial, the presence of an air pocket is a critical determinant of survival, and its absence would designate a victim as expectant. When burial time is less than 35 minutes or the time is unknown and the airway is obstructed by impacted material such as snow or by 


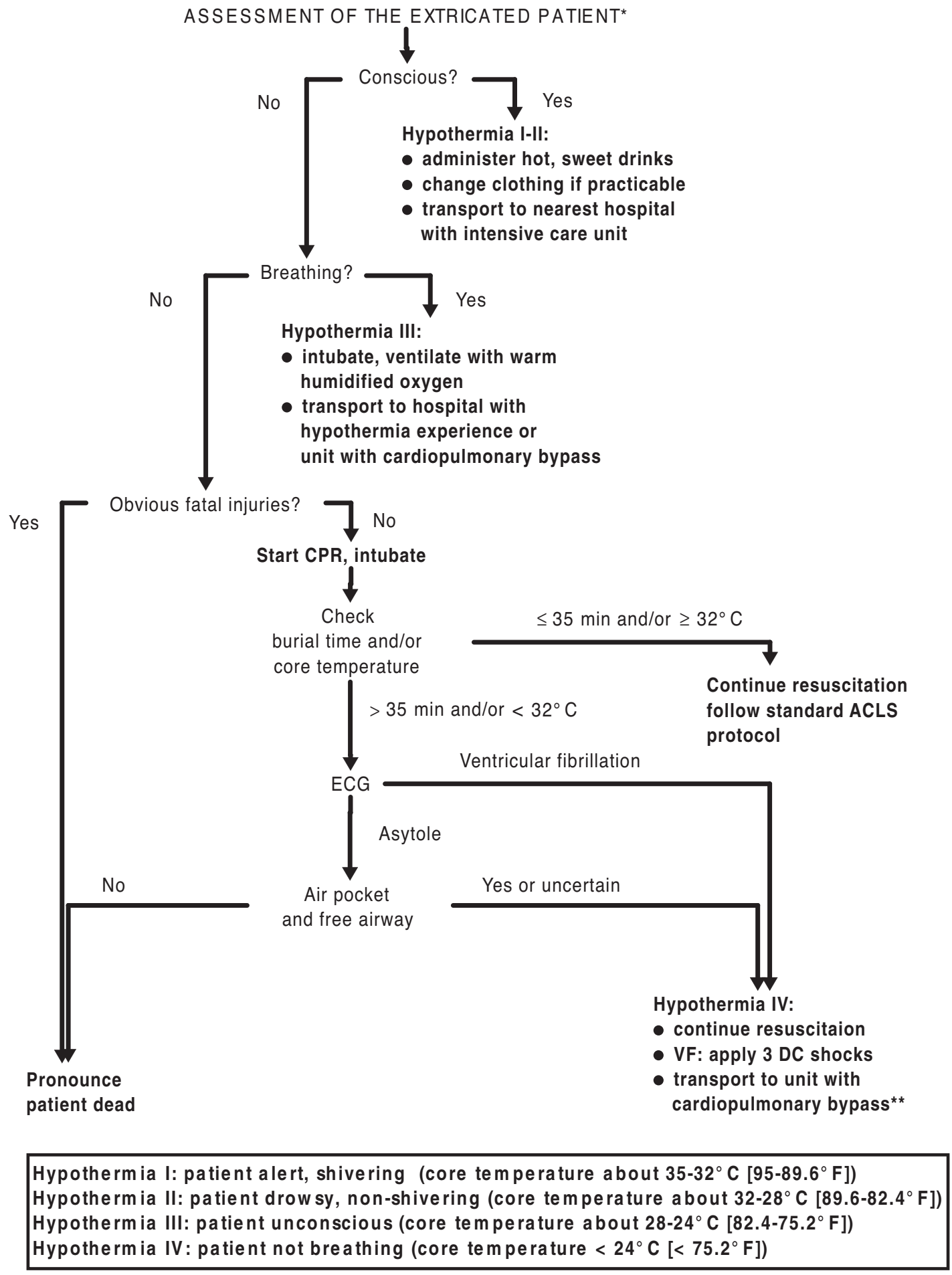

Figure 1. Algorithm for on-site management of avalanche victims. Staging of hypothermia according to Swiss Society of Mountain Medicine guidelines. *Transport to nearest hospital for serum potassium measurement if hospitalization in a specialist unit with cardiopulmonary bypass facilities is not logistically possible. Source: Reprinted from Ref. 18 with permission.

occluding posture, then the airway should be cleared, repositioned, and opened before the breathing is reassessed.
The ability to obey simple commands, the presence of a radial pulse, and heart rate are also incorporated as triage factors, as in other mass casualty triage algo- 
Table. Pattern and severity of injury in 105 avalanche victims ${ }^{a}$

Type of injury

Occurrence $(n)^{b}$

Asphyxia - fatal

33

Cervical spine fracture with

2

dislocation - fatal

Hypothermia - fatal

Extremity trauma

20

Chest trauma

18

Spine fracture

Cerebral trauma

7

Abdominal trauma

Pelvic fracture

Minor or no injury

2

1

1

21

${ }^{a}$ Source: Reprinted with permission. ${ }^{4}$

${ }^{b}$ The occurrence of these injuries distributed among the 105 victims in this study.

rithms. ${ }^{12,15,16}$ Capillary refill will be unreliable with cold exposure, and was avoided. ${ }^{30}$ If there is doubt at any decision step, such as total burial time or obvious fatal injury, the decision must be made in a conservative manner to proceed with additional evaluation of the victim. The AvSORT algorithm is designed to assign many patients to the "immediate" hospital disposition category in order to overtriage victims by the appropriate $50 \% .{ }^{13}$ Patients in the immediate category should receive priority first aid and transport out of the field and should demand more initial attention than those in the urgent category, who subsequently receive priority for treatment and transport over delayed category patients. Patients in the expectant category should only be reassessed after all other victims are attended. When manpower is increased, then further treatment of expectant victims may be considered. Formal pronouncement of death and notification of legal authorities should be performed according to procedures established in the particular jurisdiction.

Very importantly, the proposed triage algorithm does not depend on medical equipment and is designed to be simple to use in a stressful situation. It might be printed as a quick reference card to be placed in first responders rescue kits.

The 2008-2009 season was marred by an above-average avalanche fatality rate in North America with 54 deaths. ${ }^{1}$ Twenty-three of the 40 fatal incidents involved multiple individuals being caught, injured, and/or buried. The number of individuals caught in these fatal avalanches ranged from 1 to 11 individuals. Research has shown that in $26 \%$ of avalanche incidents where complete burials occurred, there were 4 or more complete burials in the party. ${ }^{7}$

The best chance for survival is for companion rescue, either by members of the same party or nearby parties, as the initial survival rate of $92 \%$ at 15 minutes of burial time plummets to only $30 \%$ at 35 minutes. ${ }^{29}$ Recovery times include location of the victim, best with an avalanche transceiver, in addition to shoveling heavy consolidated avalanche debris down to the victim and subsequent extrication from the burial hole. Deeper burials are associated with longer and more difficult extraction. Victims buried deeper than $200 \mathrm{~cm}$ have a probability of survival of only $10 \%$ compared with $80 \%$ for those buried less than $50 \mathrm{~cm}^{24}$

The inevitable delay for organized rescue, provided by a trained rescue team that is off-site, is believed responsible for the limited increase in survival despite improvements in rescue and medical care. ${ }^{29}$

The AvSORT algorithm is designed to improve avalanche victims' outcomes in a mass casualty incident by offering only the simple lifesaving interventions of opening airways and stopping external bleeding to already severely hypoxic or injured victims but allowing first responders to recover other victims who may have better chances of survival. Rescuers will also have started to triage for evacuation to appropriate facilities when transport becomes available. Using simple criteria will reduce confusion and allow direction in the potentially chaotic circumstance of a large avalanche rescue, especially with respect to the final destination of seriously injured victims. The importance of appropriate evacuation decisions is demonstrated by the finding that direct transport of more severely injured patients to an accredited trauma center has been shown to reduce morbidity and mortality. ${ }^{14,31}$ In some circumstances, due to transportation limitations, individuals may be transported to the nearest health care facility for stabilization and initial treatment until further transport can be arranged.

Mass casualty avalanche incidents not uncommonly overwhelm first responders that are limited to a few individuals and that have to manage a large number of victims forcing rescuers to focus on victim recovery efforts with limited initial resuscitation opportunities. ${ }^{7,8,27}$ As there may be no way of knowing the injury status of buried individuals, rescuers must assume that some may be buried with minor injuries but are in danger of death from asphyxiation and need prompt extraction. Thus, it would be for the greater benefit of the whole to extract potential survivors that risk asphyxia while buried rather than engage in protracted resuscitation on probable nonsurvivors. This assumption is based on the critical element of time of burial reflected in the well-established survival curve. ${ }^{29}$ Rescuers may be forced to make tactical triage decisions, incorporating such factors as relative burial depths, measured by transceiver signal strengths as well as probing, that may override initial extensive resuscitative measures. Additionally, rescuers need to ensure their own safety, and the risk of being buried in subsequent avalanches may preclude comprehensive resuscitation. ${ }^{32,33}$ 


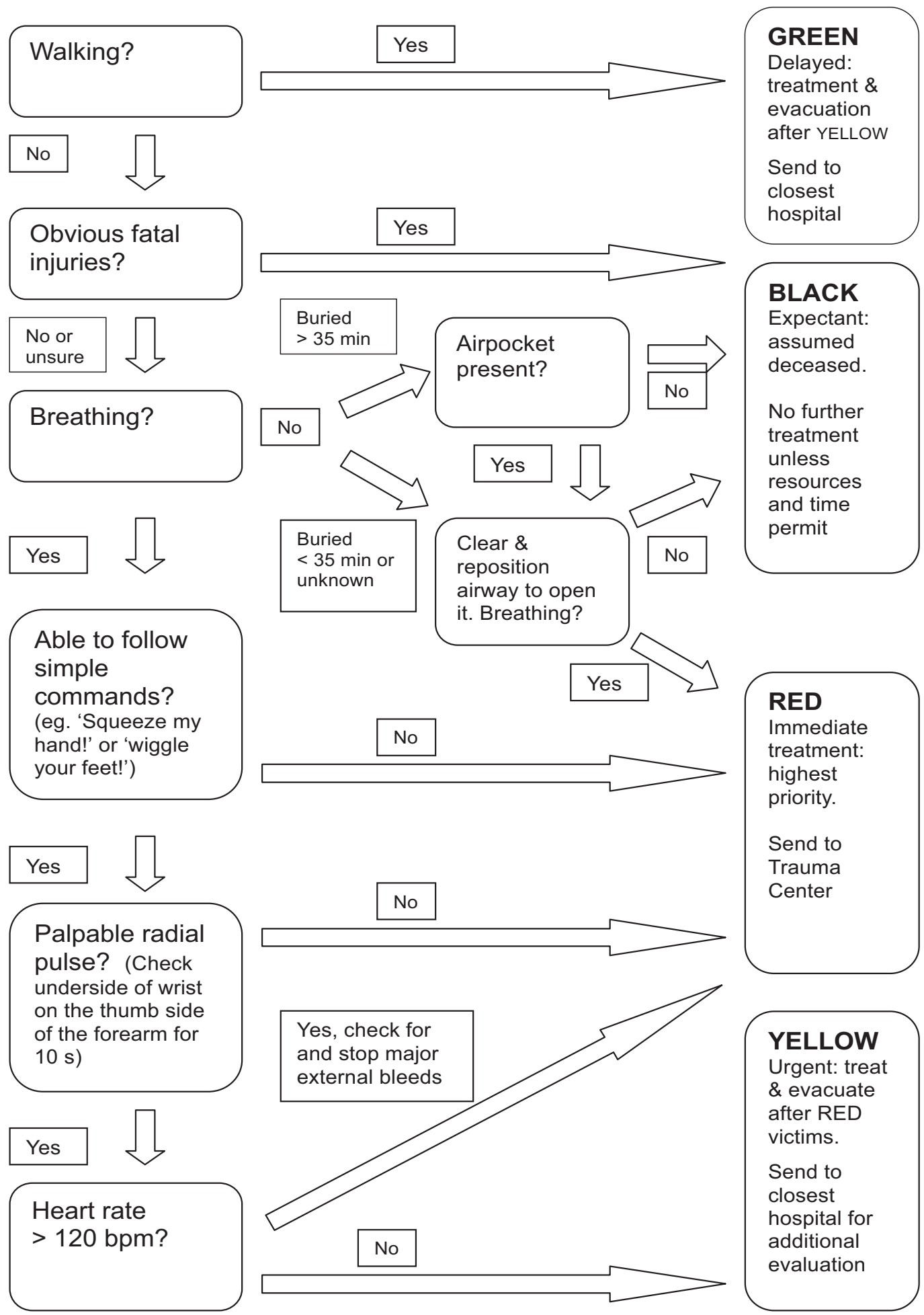

Figure 2. The Avalanche Survival Optimizing Rescue Triage (AvSORT) algorithm for the management of multiple burials in an avalanche to be used as an initial triage tool when needs exceed resources. Further treatment within the scope of rescuer ability with standard first aid principles may be warranted upon extraction and evaluation of all victims. 


\section{Limitations}

While our algorithm is designed for the initial management of mass casualty avalanche incidents when manpower is overwhelmed, it is not designed for situations where resources allow for standard resuscitation and treatment of all extracted individuals, such as in the ICAR MedCom ALS algorithm. Each avalanche incident is unique in the mix of numbers of buried, types of injuries, and abilities of first responders as well as dynamic changes in any layered response, therefore no one static system will handle all evolving situations appropriately. We propose the AvSORT algorithm to help guide rescuers to triage victims efficiently thereby optimizing survival. We anticipate the AvSORT algorithm will be used in conjunction with appropriate avalanche training, equipment, and common sense to adequately manage avalanche risk and safely execute a rescue.

Our proposed AvSORT algorithm is based on established concepts in avalanche and mass casualty triage medicine but has not been subjected to formal case-based study for this specific incident type. Further prospective research into the performance of this algorithm is needed to validate its efficacy in the field.

\section{Acknowledgments}

We wish to thank Ken Wylie and the Canadian Avalanche Association for their input and advice in this project.

\section{References}

1. Avalanche.org. Avalanche accidents database. Available at: http://avalanche.org/accidents.php. Accessed May 13, 2009.

2. Page C, Atkins D, Shockley L, Yaron M. Avalanche deaths in the United States: a 45-year analysis. Wilderness Environ Med. 1999;10:145-151.

3. Van Tilburg C. In-area and backcountry snowboarding: medical and safety aspects. Wilderness Environ Med. 2000;11:102-108.

4. Hohlrieder M, Brugger H, Schubert HM, Palvic M, Ellerton J, Mair P. Pattern and severity of injury in avalanche victims. High Alt Med Biol. 2007;8:56-61.

5. Brugger H, Durrer B, Adler-Kastner L, Falk M, Tschirky F. Field management of avalanche victims. Resuscitation. 2001;51:7-15.

6. Sasser S. Field triage in disasters. Prehosp Emerg Care. 2006;10:322-323.

7. Genswein M, Thorvaldsdottir S, Zweifel B. Remote reverse triage in avalanche rescue. Proceedings of the International Snow Science Workshop. September 21-27, 2008; Whistler, BC, Canada; Avalanche-Research.com, Olympic
Valley, CA; 2008. Available at: http://avalanche-research. com/site/dynamic_library.asp?flv=8/issw_sep22_2008_ 1340library.flv\&logID=148. Accessed April 14, 2009.

8. Stethem C, Piche M. Winter 2003 in Southern BC perspective, recognition, management. Proceedings of the International Snow Science Workshop. September 19-24, 2004; Jackson Hole, WY; International Snow Science Workshop American Avalanche Institute, Wilson, WY; 2004. Available at: www.avalanche.org/ issw2004/issw_ previous/2006/proceedings/data/papers/117.pdf. Accessed August 18, 2009.

9. Penniman D, Baumann F. The SME avalanche tragedy of January 20, 2003: a summary of the data. Proceedings of the International Snow Science Workshop. September 19-24, 2004; Jackson Hole, WY; 2004.

10. Boyd J, Wylie K. Avalanche triage. Avalanche Canada. (Revelstoke). Canadian Avalanche Association. 2008;83: 64-67.

11. Garner A, Lee A, Harrison K, Schultz C. Comparative analysis of multiple-casualty incident triage algorithms. Ann Emerg Med. 2001;38:541-548.

12. Waeckerle JF. Disaster planning and response. $N$ Engl J Med. 1991;324:815-821.

13. Kennedy K, Aghababian RV, Gans L, Lewis CP. Triage: techniques and applications in decision making. Ann Emerg Med. 1996;28:136-144.

14. Sampalis JS, Denis R, Frechette P, Brown R, Fleiszer D, Mulder D. Direct transport to tertiary trauma centers versus transfer from lower level facilities: impact on mortality and morbidity among patients with major trauma. J Trauma. 1997;43:288-295.

15. Arnold T, Cleary V, Groth S, Hook R, Jones D, Super G. START. Newport Beach, CA: Newport Beach Fire and Marine Department; 1994.

16. Hodgetts TJ, Porter C, eds. Major Incident Medical Management and Support: The Practical Approach. 2nd ed. London, UK: BMJ Publishing Group; 2002.

17. Benson M, Koenig KL, Schultz CH. Disaster triage: START, then SAVE—a new method of dynamic triage for victims of a catastrophic earthquake. Prehosp Disaster Med. 1996;11:117-124.

18. Tomm I. eTraining for Mountain Operations. Avalanche Canada. (Revelstoke). Canadian Avalanche Association. 2007;80:14-16.

19. MacKersie RC. Field triage, and the fragile supply of 'optimal resources' for the care of the injured patient. Prehosp Emerg Care. 2006;10:347-350.

20. American College of Surgeons Committee on Trauma. Advanced Trauma Life Support for Doctors: Student Course Manual. 8th ed. Chicago, IL: American College of Surgeons; 2002.

21. Brugger H, Durrer, B. On-site treatment of avalanche victims ICAR-MEDCOM-recommendation. High Alt Med Biol. 2002;4:421-424.

22. Berghold, F. Death in an avalanche-emergency medicine gleanings of the avalanche catastrophe on 28 March 2000 
in the "Hohe Tauern" [in German]. Der Notarzt. 2001; 17:182-185.

23. Johnson SM, Johnson AC, Barton R. Avalanche trauma and closed head injury: adding insult to injury. Wilderness Environ Med. 2001;12:244-247.

24. Jamieson B, Geldsetzer T. Trends and patterns in avalanche accidents. In: Avalanche Accidents in Canada Volume 4 1984-1996. Revelstoke, BC, Canada: Canadian Avalanche Association; 1996:7-20.

25. Grossman MD, Saffle JR, Thomas F, Tremper B. Avalanche trauma. J Trauma. 1989;29:1705-1709.

26. McIntosh SE, Grissom CK, Olivares CR, Kim HS, Trember B. Cause of death in avalanche fatalities. Wilderness Environ Med. 2007;18:293-297.

27. Stalsberg H, Albretsen C, Gilbert M, et al. Mechanism of death in avalanche victims. Virchows Arch A Pathol Anat Histopathol. 1989;414:415-422.

28. Brugger H, Sumann G, Meister R, et al. Hypoxia and hypercapnia during respiration into an artificial air pocket in snow: implications for avalanche survival. Resuscitation. 2003;58:81-88.
29. Falk M, Brugger H, Adler-Kastner L. Avalanche survival chances. Nature. 1994;368:21.

30. Anderson B, Kelly AM, Kerr D, Clooney M, Jolley D. Impact of patient and environmental factors on capillary refill time in adults. Am J Emerg Med. 2008;26: 62-65.

31. MacKenzie E, Rivara F, Jurkovich GJ, et al. A national evaluation of the effect of trauma-center care on mortality. N Engl J Med. 2006;354:366-378.

32. Kristensen K, Genswein M, Atkins D. Risk and avalanche rescue. Proceedings of the International Snow Science Workshop. September 21-27, 2008; Whistler, BC, Canada; Avalanche-Research.com, Olympic Valley, CA; 2008. Available at: http://avalanche-research.com/site/ dynamic_library.asp?flv=8/issw_sep26_2008_1655library. flv\&logID =195. Accessed April 14, 2009.

33. Hohlrieder M, Thaler S, Wuertl W, et al. Rescue missions for totally buried avalanche victims: conclusions from twelve years of experience. High Alt Med Biol. 2008;9: $229-233$. 